\title{
Transverse coherent instability of a bunch in a rectangular potential well
}

\author{
V. Balbekov* \\ Fermi National Accelerator Laboratory, P.O. Box 500, Batavia, Illinois 60510, USA
}

(Received 4 April 2006; published 21 June 2006)

\begin{abstract}
A theory of transverse instability of a bunch in a rectangular potential well is developed. A series of equations adequately describing the instability is derived and solved both analytically and numerically. The dependence of the instability growth rate and threshold on bunch factor is investigated for various beam coupling impedances. The theory is applied to the Fermilab recycler ring.
\end{abstract}

DOI: $10.1103 /$ PhysRevSTAB.9.064401

PACS numbers: 29.20.-c, 29.27.Bd

\section{INTRODUCTION}

The Fermilab recycler is an antiproton storage ring with stochastic and electron cooling [1]. Transverse resistive wall instability is observed in the ring at intensity several $\times 10^{11} \bar{p}$ and relatively small phase volume of the bunch. A digital instability damper with high order filter is used increasing achievable phase density by a factor $\sim 2$ [2].

A distinctive feature of the recycler is the rf system which can create a series of rectangular pulses (other possibilities are not considered here) [1]. A bunch of several microseconds long is kept in almost rectangular potential well which arises between two pulses of alternating polarity (barriers). Synchrotron frequency is very low in such a bucket (typically several $\mathrm{Hz}$ ) having a $100 \%$ spread. That is why the traditional theory of bunched beam transverse instability is not applicable, being based mostly on a model of linear synchrotron oscillations and on an approximation of uncoupled multipoles [3].

To the author's knowledge, the square potential well model was first used in Ref. [4] to demonstrate a suppression of the transverse instability by space charge tune shift. However, not sufficiently realistic air bag longitudinal phase space distribution was applied then. A more advanced model was developed in Ref. [5] to consider an electron cloud driven instability. These results cannot be directly applied to the recycler.

The first theoretical analysis of resistive wall instability in the recycler was published in Ref. [6]. It was shown that dependence of the instability growth rate on bunch factor is rather moderate, and a coasting beam model was used to find the instability threshold.

A more detailed investigation was performed in Ref. [7] where several impedances of different types were examined, including space charge and instability damper contributions. The problem was treated in terms of an effective impedance. It was shown that its real part (which is responsible for the instability) increases as the bunch shortening not faster than its imaginary part. The permissibility

*Electronic address: balbekov@fnal.gov of a coasting beam model for calculation of the instability threshold was confirmed by this.

However, a dependence of the growth rate or the effective impedance on a bunch factor was not established thoroughly in the mentioned articles. The basic challenge is a numerical calculation of high order eigenvalues of large matrices. An alternative method developed in this paper does not require the use of such cumbersome matrices, and allows one to investigate the growth rate and threshold of very high eigenmodes. This is especially important for systems with an instability damper, where these modes are most unstable.

We will consider a single bunch neglecting the penetration of particles into the barriers. Such an assumption is well suited for the Fermilab recycler at the beam storage and cooling, when depth of the penetration is 1-2 orders of magnitude less than the bunch length [1]. By this model, the absolute value of the particle longitudinal momentum is an integral of motion in the bunch rest frame, like action, and direction of the motion alternates instantly. These properties somewhat liken the barrier bucket to a coasting beam, and makes the soluble problem in the end. Betatron oscillations are taken to be linear, because nonlinearity of the external field is very small in the recycler, and nonlinearity of the space charge field does not affect the transverse oscillations of the beam center [8].

In Secs. II and III, the known equations describing a bunched beam transverse instability are adapted for the rectangular potential well model. A conception of an effective impedance is introduced in Sec. IV for the zeroslippage factor, and developed in Sec. V to go beyond the scope of this approximation. On this basis, the general investigation of the instability growth rate and threshold is performed in Sec. VI and VII to be applied to the Fermilab recycler in Sec. VIII. The conclusions are summarized in Sec. IX.

\section{BASIC EQUATIONS}

Let us consider the transverse dipole moment of a beam in its rest frame: $D(\theta)=\sum_{k} D_{k} \exp (i k \theta)$ where $\theta$ is longitudinal coordinate (azimuth), and dependence on time is 
presumed to be given by factor $\exp (-i \omega t)$. Then the Fourier coefficients $D_{k}$ satisfy the following series of equations $[3,7]$ :

$$
D_{k}=\frac{i r_{0} \omega_{0} N}{2 \pi \gamma Q_{0} Z_{0}} \sum_{l} C_{k, l}(\omega) Z_{l}(\omega) D_{l},
$$

where $r_{0}=e^{2} / m c^{2}$ (about $1.535 \times 10^{-16} \mathrm{~cm}$ for protons), $Z_{0}=4 \pi / c \simeq 376.7 \Omega, N$ is the beam intensity, $\omega_{0}$ and $Q_{0}$ are central angular velocity and betatron tune, respectively. Factors $Z_{l}(\omega)$ can be represented in terms of transverse beam coupling impedance in the laboratory frame, or in terms of the corresponding wake field [9]:

$$
Z_{k}(\omega)=Z\left(\omega+k \omega_{0}\right)=i \int_{0}^{\infty} W(\theta) \exp \left(i\left[k+\frac{\omega}{\omega_{0}}\right] \theta\right) d \theta .
$$

The general formula for $C_{k, l}(\omega)$ is

$$
C_{k, l}(\omega)=\sum_{m} \int_{0}^{\infty} \frac{I_{m, k}(\nu, \epsilon) I_{m, l}^{*}(\nu, \epsilon) \mathcal{F}(\epsilon) d \epsilon}{\omega+\omega_{0} Q_{0}-m \Omega(\epsilon)},
$$

where $\epsilon$ is longitudinal action and $\mathcal{F}(\epsilon)$ is the corresponding normalized distribution function, $\Omega(\epsilon)$ is synchrotron frequency, and $\nu=Q_{0}-\xi / \eta, \xi$ and $\eta$ are the machine chromaticity and slippage factor, respectively. Form factors $I_{m, k}(\nu, \epsilon)$ are the coefficients of expansion of a planar wave in a series of multipoles:

$$
I_{m, k}(\nu, \epsilon)=\frac{1}{2 \pi} \int_{-\pi}^{\pi} \exp \{i m \phi-i[k-\nu] \theta(\epsilon, \phi)\} d \phi,
$$

where the particle azimuth $\theta$ should be presented as a function of synchrotron action and phase.

\section{RECTANGULAR POTENTIAL WELL}

Now we have to apply these general formulas to the particles in a rectangular potential well of length $2 \pi B$ where $B$ is the bunch factor. Then Eq. (4) gives

$$
I_{m, k}(\nu)=2 i B[k-\nu] \frac{1-\exp \{i \pi(2 B[k-\nu]-m)\}}{\pi\left(4 B^{2}[k-\nu]^{2}-m^{2}\right)},
$$

and the synchrotron frequency is

$$
\Omega=\frac{\omega_{0}|\epsilon \eta|}{8 \pi p_{0} B^{2}}=\frac{\omega_{0}\left|\left(p-p_{0}\right) \eta\right|}{2 p_{0} B},
$$

where $p$ is the particle momentum in the laboratory frame and $p_{0}$ is the central momentum of the beam [7]. Therefore, one can represent coefficients (3) of series (1) in the form

$$
\begin{aligned}
C_{k, l}(\omega)= & \sum_{m} I_{m, k}(\nu) I_{m, l}^{*}(\nu) \\
& \times \int_{-\infty}^{\infty} \frac{F(p) d p}{\omega+\omega_{0} Q_{0}-m \omega_{0} \eta\left[p-p_{0}\right] /\left[2 B p_{0}\right]},
\end{aligned}
$$

where $F(p)$ is the normalized distribution function on momentum.

Because the factors $I_{m, k}$ do not depend on action now, another form of series (1) can be proposed:

$$
\begin{aligned}
X_{m}= & \frac{i r_{0} \omega_{0} N}{2 \pi \gamma Q_{0} Z_{0}} \sum_{n} Z_{m, n}(\omega) X_{n} \\
& \times \int_{-\infty}^{\infty} \frac{F(p) d p}{\omega+\omega_{0} Q_{0}-n \omega_{0} \eta\left[p-p_{0}\right] /\left[2 B p_{0}\right]},
\end{aligned}
$$

where

$$
X_{m}=\sum_{k} Z_{k}(\omega) I_{m, k}^{*}(\nu) D_{k}
$$

and

$$
Z_{m, n}(\omega)=\sum_{k} Z_{k}(\omega) I_{m, k}^{*}(\nu) I_{n, k}(\nu) .
$$

This form will be largely used for the analysis. Note that variables $X_{m}$ can be treated as amplitudes of the longitudinal multipoles in the bunch spectrum.

\section{ZERO-SLIPPAGE LIMIT}

As it was mentioned above, the synchrotron frequency is typically very low in the recycler ring. To be specific, it is small in comparison with the space charge driven tune shift which is another characteristic frequency of the problem. The statement would be wrong only for very short bunches. However, we will not consider this case because the penetration of the particles into the barriers becomes essential earlier, in practice. Therefore, the limit $\Omega \rightarrow 0$ can be taken as a reasonable first approximation.

The corresponding limiting process should not be performed by decreasing of the distribution width, because the effect of chromaticity would also be lost. It is necessary to proceed to the limit $\eta \rightarrow 0$ taking into account that $\nu \rightarrow \infty$ in this case. Then the dispersion equation following from series (1) is

$$
1=\frac{i r_{0} \omega_{0} N Z_{M}^{(e f)}(\omega)}{2 \pi \gamma Q_{0} Z_{0}} \int_{-\infty}^{\infty} \frac{F(p) d p}{\omega+\omega_{0} Q(p)},
$$

where $Q(p)=Q_{0}+\xi\left[p-p_{0}\right] / p_{0}$ is the momentum dependent betatron frequency [7]. The equation includes the effective impedance which is the $M$ th eigenvalue of the series of equations:

$$
Z_{M}^{(e f)}(\omega) D_{k}=\sum_{l} \rho_{k-l} Z_{l}(\omega) D_{l}
$$

where 


$$
\rho_{k}=\frac{\sin (\pi B k)}{\pi B k} \exp (-i \pi B k)
$$

are Fourier coefficients of the normalized linear density of the beam: $\rho(\theta)=1 / B$ at $0<\theta<2 \pi B$. It is easy to see that $D_{k}=\delta_{k, M}$ and $Z_{M}^{(e f)}(\omega)=Z_{M}(\omega)$ at $B=1$.

It can also be shown that, for the rectangular potential well,

$$
\rho_{k-l}=\sum_{m} I_{m, k}(\nu) I_{m, l}^{*}(\nu) .
$$

This relation allows one to obtain another form of series (12) corresponding to Eq. (8):

$$
Z_{M}^{(e f)}(\omega) X_{m}=\sum_{n} Z_{m, n}(\omega) X_{n}
$$

One more form can be obtained by an inverse Fourier transformation of series (12) resulting in the integral equation:

$$
Z_{M}^{(e f)} D(\theta)=i \rho(\theta) \int_{0}^{\infty} D\left(\theta+\theta^{\prime}\right) W\left(\theta^{\prime}\right) \exp \left(\frac{i \omega \theta^{\prime}}{\omega_{0}}\right) d \theta^{\prime},
$$

where $W(\theta)$ and $Z(\omega)$ are connected by Laplace transformation (2). A similar equation was applied earlier for an analysis of resistive wall instability in the recycler [6].

\section{EFFECTIVE IMPEDANCE}

Several specific examples of effective impedance are considered below. Series (15) is used being the most convenient for numerical calculations. Its main advantage is that the matrix $Z_{m, n}$ is nearly diagonal, which makes it possible to calculate eigenvalues by use of its relatively small fragments. Note that the effective impedance does not depend on which value of $\nu$ is taken in Eq. (10), because its change produces a unitary transformation of the matrix. In fact, all of the calculations below have done at $\nu=0$. In addition, it is taken into account that $Z_{m, n}=$ $Z_{-m, n}=Z_{m,-n}$ to reduce series (15) to the form

$$
Z_{M}^{(e f)}(\omega) X_{m}=\sum_{n=0}^{\infty} Z_{m, n}(\omega) X_{n}
$$

where

$$
Z_{m, n}(\omega)=\sum_{k} Z_{k}(\omega) I_{m, k}^{*}(0) I_{n, k}(0) \times \begin{cases}1 & \text { at } n=0 \\ 2 & \text { at } n>0\end{cases}
$$

\section{A. Exponential wake}

Let us consider the wake field,

$$
W(\theta)=-Z^{(\exp )} \frac{\omega_{f}}{\omega_{0}} \exp \left(-\frac{\omega_{f} \theta}{\omega_{0}}\right)
$$

and corresponding impedance in frequency domain,

$$
Z(\hat{\omega})=\frac{Z^{(\exp )}}{\hat{\omega} / \omega_{f}+i},
$$

where $1 / \omega_{f}$ is the wake field decay time, and the symbol "hat" marks the laboratory frame. The example is important because an analytical solution of the problem is possible in this case. In terms of Eq. (16), the eigenfunctions and corresponding eigenvalues are

$$
\begin{aligned}
D(\theta)= & \rho(\theta) \exp \left(i K \theta+\left[i \frac{\omega+K \omega_{0}}{\omega_{0}}-\frac{\omega_{f}}{\omega_{0}}\right]\right. \\
& \left.\times\left[\int_{0}^{\theta} \rho\left(\theta^{\prime}\right) d \theta^{\prime}-\theta^{\prime}\right]\right)
\end{aligned}
$$

and

$$
Z_{K}^{(e f)}(\omega)=Z_{K}(\omega)=\frac{Z^{(\exp )}}{\left(\omega+K \omega_{0}\right) / \omega_{f}+i} .
$$

It is most remarkable that these eigenvalues do not depend on the bunch factor.

The eigenvalues of series (17) with a positive real part are represented in Fig. 1 at $Z^{(\exp )}=1, \omega_{f}=\omega_{0}$, being numerically calculated by the following method. At any $B$, the first 20 of them are obtained with the help of the $100 \times 100$ matrix $Z_{m, n}$ starting from the 0th multipole. Each other point is the first eigenvalue of a $10 \times 10$ matrix starting from multipole $50,100, \ldots, 2000$. The number $M$ is defined as the index of the highest power multipole in the spectrum of the eigenmode. Note that only odd $M$ appear in Fig. 1 because real parts of the eigenvalues are found to be negative otherwise.

The values $B=1,1 / 2,1 / 4,1 / 8$, and $1 / 16$ was actually taken for the numerical solution providing practically the same results. Because they are indistinguishable in the

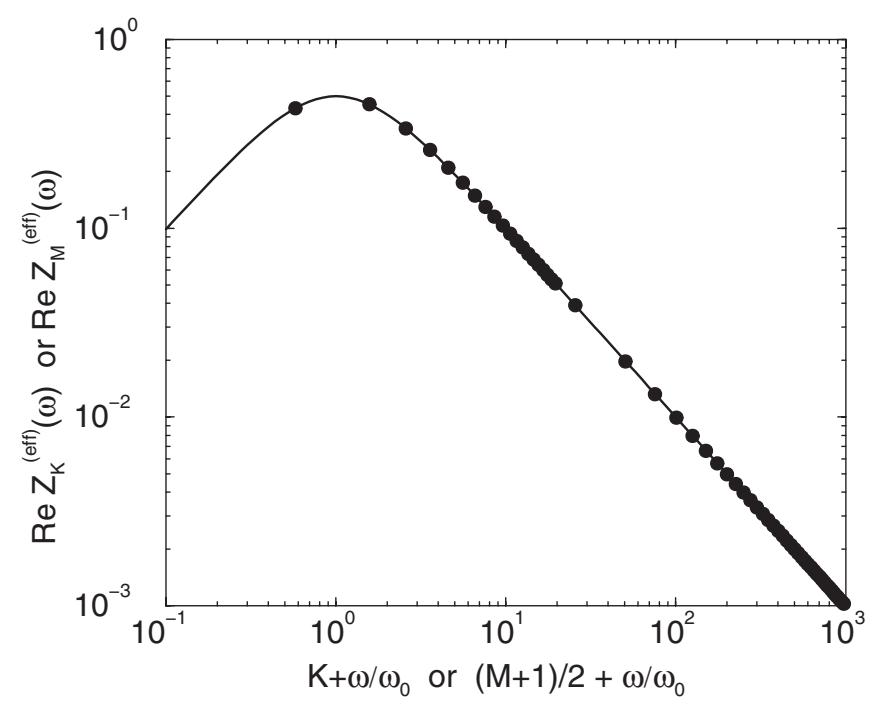

FIG. 1. Real part of exponential wake effective impedance. Circles-numerical solutions at $\omega / \omega_{0}=-0.425$, and any $B$; solid line - analytical solution (22). 
graph, the only symbol is used to represent the eigenvalues at any $B$. According to Eq. (22), all of them should be located on the solid line plotted in Fig. 1. This is so indeed, and there is a perfect agreement of numerical and analytical solutions at the relation

$$
K=\frac{M+1}{2} .
$$

It is seen from Fig. 1 that practically the same-and valid - results can be obtained by using matrices $10 \times 10$ or $100 \times 100$. It means that any eigenmode contains a rather small number of essential multipoles, a statement which does not conflict with Eq. (21). It is believed that such a narrowness of the spectrum results from the properties of motion in the rectangular well, and also is inherent in other impedances. If so, it facilitates a numerical solution of the problem at least, because the use of lowdimension matrices is admissible for the calculations (this property was noted in Ref. [5]). What is more important, this property allows one to go beyond the scope of the zero-slippage approximation at the calculation of the instability threshold, a thesis which will be developed in Sec. VII.

To test the hypothesis, the same technique is applied below to 3 practically important impedances. Because analytical solutions are not achievable in these cases at $B \neq 1$, different order matrices (up to $100 \times 100$ ) were used for the numerical solution. The results obtained with the help of the $10 \times 10$ matrix are taken for plotting in Fig. 2-4; however, any other considered matrices provide practically the same results. Physically very clear fits are shown in the figures as well, a coincidence with which the calculation should be considered as an additional argument in a favor of the checked assumption.

\section{B. Resistive wall impedance}

Taking the resistive wall impedance in the form

$$
Z(\hat{\omega})=Z^{(r w)}[\operatorname{sgn}(\hat{\omega})-i] \sqrt{\left|\frac{\omega_{0}}{\hat{\omega}}\right|}
$$

it is possible to obtain corresponding effective impedance which is presented in Fig. 2 at $Z^{(r w)}=1$. The following formula is used to fit the results:

$$
\begin{aligned}
Z_{M}^{(e f)}(\omega) & =\frac{1}{\sqrt{B}} Z\left(\omega+\frac{\omega_{0}[M+1]}{2}\right) \\
& =\frac{1}{B} Z\left(\frac{\omega}{B}+\frac{\omega_{0}[M+1]}{2 B}\right) .
\end{aligned}
$$

At $B=1$, the fit coincides with an analytical solution of Eq. (12) if relation (23) is also applied. At arbitrary $B$ and $M>\sim 10$, rather good agreement is provided by the expression:

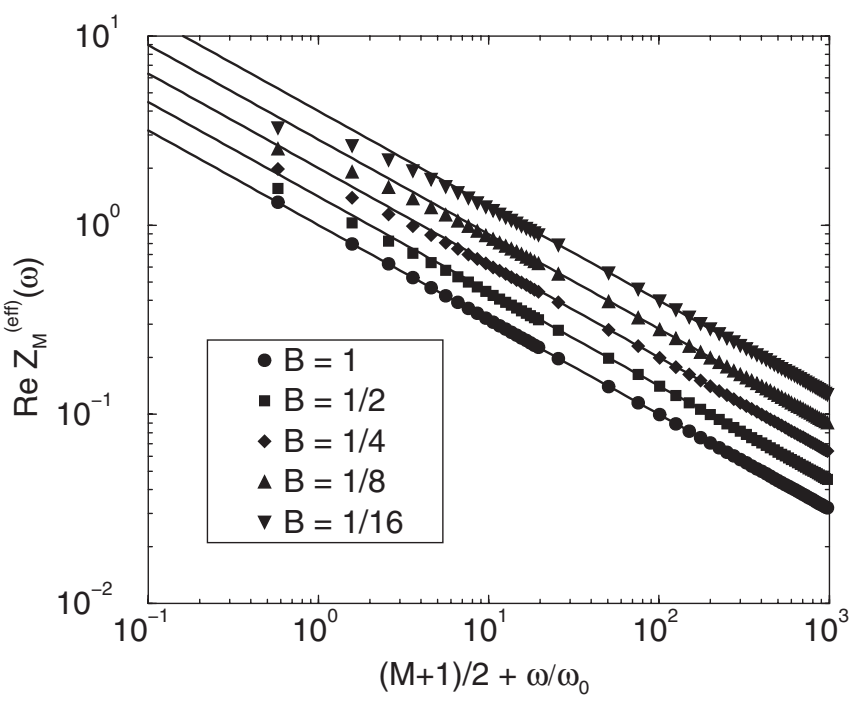

FIG. 2. Real part of resistive wall effective impedance. Solid lines represent fit (25).

$$
Z_{M}^{(e f)}(\omega) \simeq \frac{1}{B} Z\left(\frac{\omega_{0} M}{2 B}\right)
$$

However, the agreement is worse at lower $M$. In particular, a better approximation for the lowest unstable mode is

$$
Z_{1}^{(e f)} \simeq \frac{Z^{(r w)}[1-i]}{B^{1 / 3} \sqrt{K-Q_{0}}}
$$

where $K$ is the minimal integer exceeding $Q_{0}$.

\section{Resistive wall + first order damper}

At imaginary $Z^{(\exp )}$, impedance (20) represents the simplest model of an instability damper with first order $R C$ filter (imaginariness is actually provided by appropriate arrangement of pickup, kicker, and delay line). We consider it in combination with the resistive wall contribution representing the full impedance in the form

$$
Z(\hat{\omega})=Z^{(r w)}\left[[\operatorname{sgn}(\hat{\omega})-i] \sqrt{\left|\frac{\omega_{0}}{\hat{\omega}}\right|}-\frac{i G}{\hat{\omega} / \omega_{f}+i}\right]
$$

The effective impedances are calculated at $Z^{(r w)}=1$, $\omega_{f} / \omega_{0}=200$, and several $G$. Their real parts are shown in Fig. 3 in the area of rather large $M$, where positive values appear for the first time. Many of the symbols overlap here like Fig. 1. Fits obtained by means of Eqs. (26) and (28) are plotted as well, providing very good agreement for positive values. 


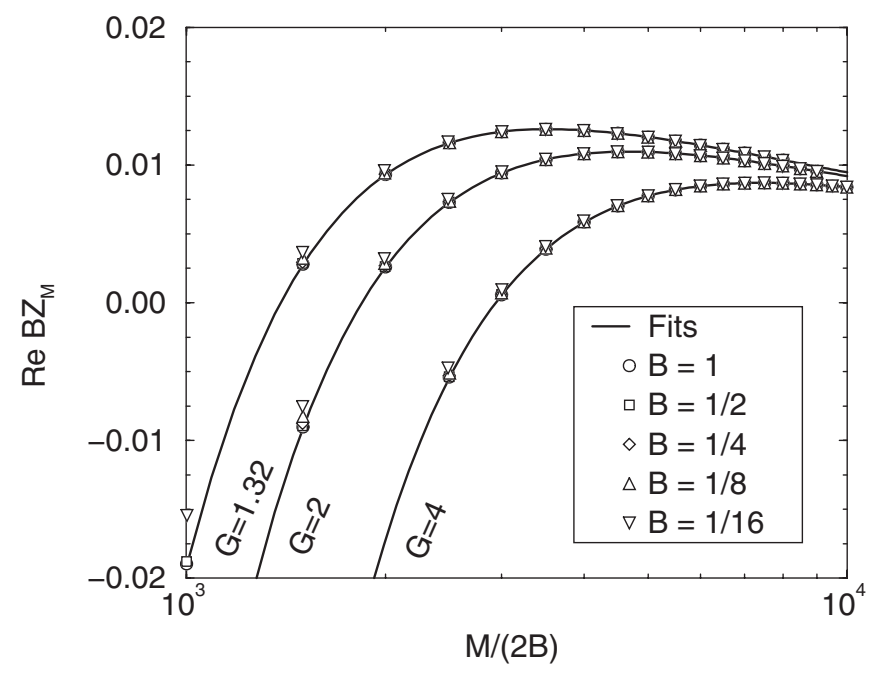

FIG. 3. Real part of resistive wall + first order damper effective impedance.

\section{Resistive wall and high order damper}

The impedance

$$
\begin{aligned}
Z(\hat{\omega})= & Z^{(r w)}\left[[\operatorname{sgn}(\hat{\omega})-i] \sqrt{\left|\frac{\omega_{0}}{\hat{\omega}}\right|}\right. \\
& -\left[\frac{\sin \left(\pi \hat{\omega} / \omega_{s}\right)}{\pi \hat{\omega} / \omega_{s}}\right]^{2}\left\{\begin{array}{ll}
G & \text { at }|\hat{\omega}|<\omega_{f} \\
0 & \text { at }|\hat{\omega}|>\omega_{f}
\end{array}\right]
\end{aligned}
$$

is considered in this subsection. At noninteger $\omega_{s} / \omega_{0}$, the addition to resistive wall part can be interpreted as a simple model of digital damper with sampling frequency $\omega_{s}$ and high order filter of bandwidth $\omega_{f}[10]$. The case $G=5 / 3$, $\omega_{s} / \omega_{0}=588.1, \omega_{f}=\omega_{s}$ is plotted in Fig. 4. Again, the numerical values are fitted very well by Eq. (26). Similar results are obtained at $\omega_{f}<\omega_{s}$ as well.

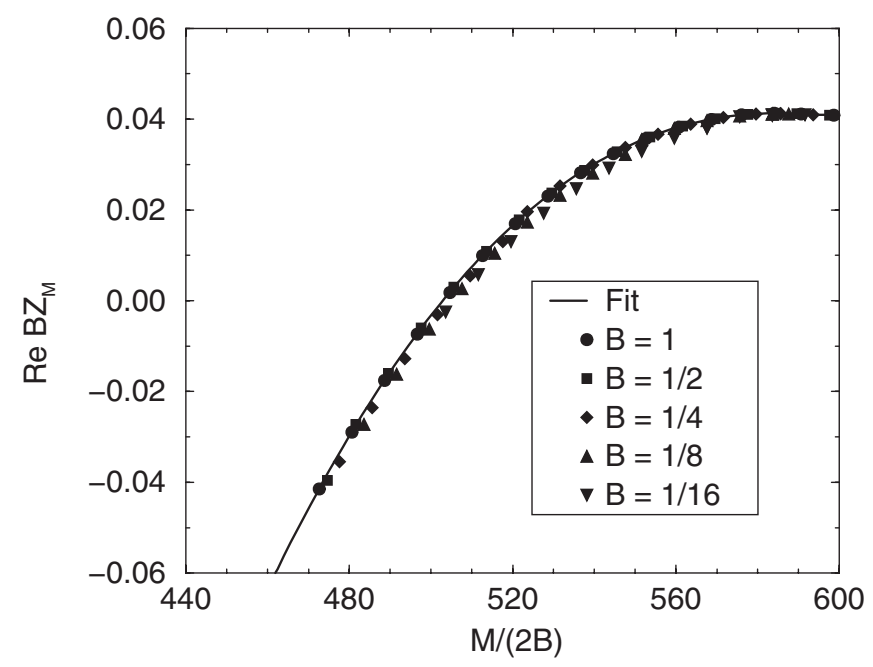

FIG. 4. Real part of resistive wall + high order damper effective impedance.

\section{THE INSTABILITY GROWTH RATE}

According to Eq. (11), at relatively small momentum spread the instability growth rate is

$$
\operatorname{Im} \omega=\frac{r_{0} \omega_{0} N}{2 \pi \gamma Q_{0} Z_{0}} Z_{M}^{(e f)}\left(-\omega_{0} Q_{0}\right) .
$$

As shown in the previous section, its dependence on bunch factor is rather diverse. For the most unstable modes, the obtained results can be summarized as: (i) exponential wake: no dependence on $B$; (ii) resistive wall: approximately $\propto B^{-1 / 3}$; (iii) resistive wall + high frequency damper: $\propto B^{-1}$.

In the last case the growth rate depends on local beam density only, which means that the instability is driven by a short-range interaction. This fact can be explained by taking into account that the bunch spectrum includes a relatively small number of high order multipoles in this case, concentrating near $M \sim 2 B \omega_{f} / \omega_{0}$. Another important point is an intimate connection of the multipoles and space harmonics due to the relation $\theta=2 B|\phi|$. As a result, the beam spectrum of any unstable mode in laboratory frame includes frequencies $\sim \omega_{f} \pm \Delta \omega$, where $\Delta \omega<$ $\sim \omega_{0} / B$ arises because of the bunching. At $\omega_{f} \gg \omega_{0} / B$, only high-frequency harmonics are present in the spectrum. Typically, they are rather quickly damped out, resulting in a suppression of long-range interaction.

Similar reasoning could be applied to any high order mode (though it is unobservable in practice). Being consistent with Eq. (26) for resistive wall instability, this statement contradicts - from the first glance - the exponential wake effective impedance (22) because the last does not depend on the bunch factor. In fact, there is no contradiction here because the statement is related to high modes, i.e., to high frequency only. Then it follows from (20) and (22):

$$
Z^{(e f)}(\omega)=Z(\omega) \propto 1 / \omega \propto Z(\omega / B) / B,
$$

in total agreement with Eq. (26).

\section{THRESHOLD OF THE INSTABILITY}

Frequency independent space charge impedance $Z=$ $-i Z^{(s c)}$ should be taken into account when the instability threshold is calculated, because it usually produces a determining effect on Landau damping. Using Eq. (18), it is easy to verify that the inclusion provides an additive contribution $-i Z^{(s c)} / B$ to all diagonal elements of the matrix $Z_{m, n}$, i.e., to all its eigenvalues. For example, the threshold of the lowest (most unstable) mode of resistive wall instability should be determined from Eq. (11), where

$$
Z_{1}^{(e f)}=\frac{Z^{(r w)}[1-i]}{B^{1 / 3} \sqrt{K-Q_{0}}}-\frac{i Z^{(s c)}}{B}
$$

and minimal $K>Q_{0}$ is applied. Absence of slippage factor 
in this case is actually immaterial, because its contribution to frequency spread would be small in comparison with chromaticity contribution.

However, the slippage can be important for the analysis of a wideband damper, because fast-modulated eigenmodes with a dominant contribution of higher multipoles would be the most unstable in this case. Fortunately, this drawback can be easily remedied due to the narrowness of the eigenmode spectrum which is established in Sec. V. It is sufficient to separate corresponding central multipoles $n=$ $\pm M$ in Eq. (8) and to retain them in all following transformations. Next, taking into account also Eq. (6) and relation $F\left(p-p_{0}\right)=F\left(p_{0}-p\right)$, the following equation can be obtained instead of Eq. (11):

$$
\begin{aligned}
1= & \frac{i r_{0} \omega_{0} N Z_{M}^{(e f)}(\omega)}{2 \pi \gamma Q_{0} Z_{0}} \\
& \times \int_{-\infty}^{\infty} \frac{F(p) d p}{\omega+\omega_{0} Q(p)+M \omega_{0} \eta\left[p-p_{0}\right] / 2 B p_{0}} .
\end{aligned}
$$

This expression can be represented in the form very similar to the coasting beam dispersion equation:

$$
1=\frac{i r_{0} \omega_{0} N Z^{(e f)}(\hat{\omega})}{2 \pi \gamma Q_{0} Z_{0}} \int_{-\infty}^{\infty} \frac{F(p) d p}{\hat{\omega}-\omega_{r}(p)[\kappa-Q(p)]},
$$

where $\omega_{r}(p)$ is angular velocity of a particle with momentum $p$ in the laboratory frame, $\hat{\omega}=\omega+\kappa \omega_{0}, \kappa=M / 2 B$. An appropriate form of the effective impedance should be used in this equation. For example, substitution of Eq. (31) allows one to find a threshold of resistive wall instability. When the higher modes are considered, Eq. (26) should be used resulting in a simple relation:

$$
Z^{(e f)}(\hat{\omega}) \simeq \frac{Z(\hat{\omega})}{B},
$$

where $Z(\hat{\omega})$ is total beam coupling impedance, including space charge contribution, resistive wall, damper, etc.

The Gaussian distribution function $F$ with dispersion $\sigma$ is considered below. Then all the solutions of Eq. (33) are stable $(\operatorname{Im} \omega \leq 0)$ at the condition

$$
\left|\frac{\operatorname{Re} \Delta \omega}{\delta \omega}\right|<f\left(\left|\frac{\operatorname{Re}(\Delta \omega)}{\operatorname{Im}(\Delta \omega)}\right|\right)=f\left(\left|\frac{\operatorname{Im}\left(Z^{(e f)}\right)}{\operatorname{Re}\left(Z^{(e f)}\right)}\right|\right),
$$

where $\Delta \omega$ is the impedance produced frequency shift without Landau damping:

$$
\Delta \omega=\frac{i r_{0} \omega_{0} N Z^{(e f)}}{2 \pi \gamma Q_{0} Z_{0}}
$$

$\delta \omega$ is the rms frequency spread due to the momentum spread:

$$
\delta \omega=\left|\xi+\eta\left[\kappa-Q_{0}\right]\right| \frac{\omega_{0} \sigma}{p_{0}},
$$

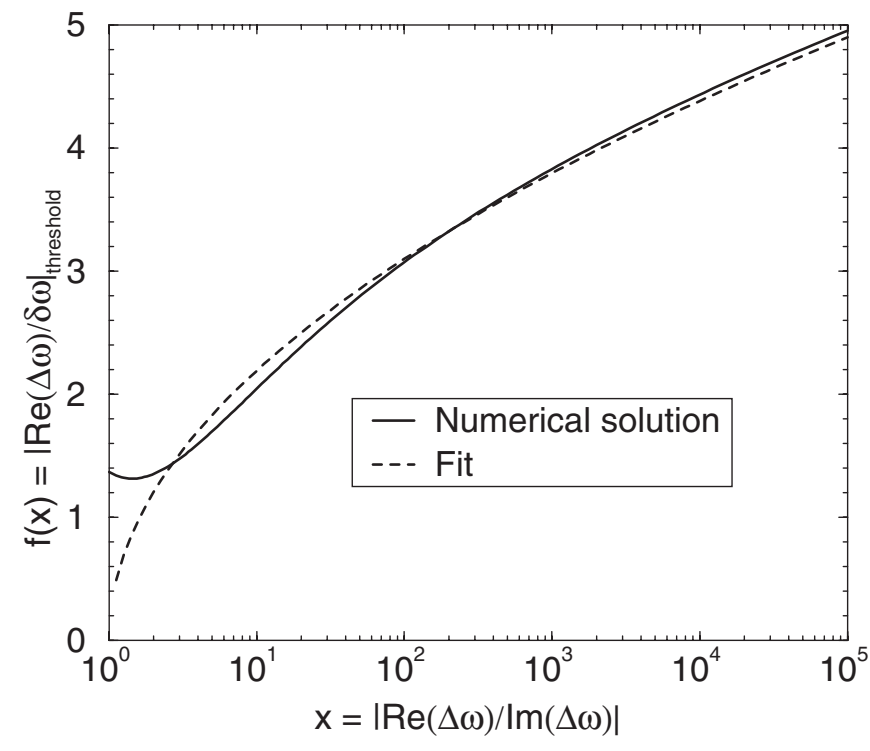

FIG. 5. Plot to calculate threshold of instability at Gaussian distribution.

and function $f$ is represented by solid line in Fig. 5. A simple fit

$$
f(x) \simeq \sqrt{4.8 \log (x)}
$$

is also plotted providing rather good approximation at $x>$ 3.

The space charge impedance often dominates among others, so that imaginary part of the total impedance significantly exceeds its real part. If the beam transverse distribution function is Gaussian, the statement can be written in the form

$$
\left|\operatorname{Re} Z^{(e f)}\right| \ll\left|\operatorname{Im} Z^{(e f)}\right| \simeq \frac{Z^{(s c)}}{B}=\frac{\pi Z_{0} Q_{0}}{4 B S_{\perp} \beta \gamma},
$$

where $S_{\perp}$ is transverse normalized rms phase volume of the beam [8]. Then stability condition (35) can be represented in the form:

$\frac{N}{S_{\|} S_{\perp}}<\frac{4 \omega_{0} \gamma\left|\xi+\left[\kappa-Q_{0}\right] \eta\right|}{\pi \beta e^{2}} f\left(\left|\frac{\pi Q_{0} Z_{0}}{4 \beta \gamma S_{\perp} B \operatorname{Re}\left(Z^{(e f)}\right)}\right|\right)$,

where

$$
S_{\|}=B C \sigma
$$

is treated as longitudinal rms phase volume of the bunch, and $C$ is the machine circumference.

\section{EXAMPLE: FERMILAB RECYCLER}

We continue the analysis taking the Fermilab recycler ring as an example with the following parameters: 
(i) $\omega_{0}=2 \pi \times 89.86 \mathrm{kHz}, \quad$ (ii) $\gamma=9.526$, (iii) $\xi=$ -(2-6), (iv) $\eta=-0.0085$, and (v) $Q_{0}=25.425$.

Then Eqs. (38) and (40) give the condition of stability:

$$
\begin{aligned}
D \equiv & \frac{N / 10^{10}}{4 S_{\|}(\mathrm{eV}-\mathrm{s}) 6 S_{\perp}(\pi-\mathrm{mm}-\mathrm{mrad})}<0.14 \mid \xi \\
& -0.0085\left[\kappa-Q_{0}\right] \mid \sqrt{\log \left|\frac{0.66 \pi Z_{0}}{S_{\perp} B \operatorname{Re}\left(Z^{(e f)}\right)}\right|},
\end{aligned}
$$

where $95 \%$ emittances are used in the definition of space phase density $D$. Some special cases are considered below.

\section{A. Resistive wall impedance}

This impedance is the main source of instability in the recycler. Characteristic parameter $Z^{(r w)} \simeq 18 \mathrm{M} \Omega / \mathrm{m}$ $[2,6,7]$ provides for the lowest (most unstable) mode:

$$
\operatorname{Re} Z_{1}^{(e f)} \simeq \frac{24(\mathrm{M} \Omega / \mathrm{m})}{B^{1 / 3}} .
$$

Because slippage is negligible in this case, Eq. (42) gives

$$
D<0.14|\xi| \sqrt{\log \left(\frac{10}{S_{\perp} B^{2 / 3}}\right)} .
$$

It means that the beam becomes more stable as shortening, though the dependence is very weak, and estimation $D<$ $\sim 0.14|\xi| \simeq 0.3 \div 0.8$ is valid at $S_{\perp} \sim 1 \pi$-mm-mrad and any reasonable $B$.

\section{B. Resistive wall + first order damper}

As it is shown in Sec. V C, at $\omega_{f} \gg \omega_{0}$ and $\kappa \gg 1$ the real part of total effective impedance is

$$
\operatorname{Re} Z^{(e f)}=\frac{18(\mathrm{M} \Omega / \mathrm{m})}{B}\left[\frac{1}{\sqrt{\kappa}}-\frac{G}{1+\omega_{0}^{2} \tau^{2} \kappa^{2}}\right] .
$$

This value becomes positive at $\kappa>\kappa_{0} \simeq\left[G / \omega_{0}^{2} \tau^{2}\right]^{2 / 3}$, but the beam still remains stable at the condition

$$
D<0.14|\xi-0.0085 \kappa| \sqrt{\log \left(\frac{14 \sqrt{\kappa}}{S_{\perp}\left[1-\left(\kappa_{0} / \kappa\right)^{3 / 2}\right]}\right)},
$$

which does not depend on the bunch factor. In dependence on $\kappa_{0}$, the right-hand part reaches a minimum (the beam becomes most unstable) at $\kappa \simeq(1.05-1.10) \kappa_{0}$. A substitution to Eq. (46) results in the expression

$$
D_{\min } \simeq 0.28\left|0.009 \kappa_{0}-\xi\right|,
$$

which provides acceptable accuracy at $S_{\perp} \simeq$
$1 \pi$-mm-mrad. For example, at $G=4$ and $\omega_{f} / \omega_{0}=200$ (18 MHz filter) $\kappa_{0} \simeq 2950$, i.e. $D_{\min } \simeq 0.28|27-\xi|=$ $8.1 \div 9.2$.

\section{Resistive wall + high order damper}

As it is shown in Sec. VD, at $\omega_{f} \gg \omega_{0}$ and $\kappa \gg 1$ the real part of the total effective impedance is

$$
\begin{aligned}
\operatorname{Re}\left(Z^{(e f)}\right)= & \frac{18(\mathrm{M} \Omega / \mathrm{m})}{B}\left[\frac{1}{\sqrt{\kappa}}-\left[\frac{\sin \left(\pi \kappa \omega_{0} / \omega_{s}\right)}{\pi \kappa \omega_{0} / \omega_{s}}\right]^{2}\right. \\
& \times\left\{\begin{array}{ll}
G & \text { at }\left|\kappa \omega_{0}\right|<\omega_{f} \\
0 & \text { at }\left|\kappa \omega_{0}\right|>\omega_{f}
\end{array}\right] .
\end{aligned}
$$

With reasonable accuracy, ultimate beam density can be found by the expression

$$
D_{\text {min }} \simeq 0.22\left|0.0086 \frac{\omega_{f}}{\omega_{0}}-\xi-0.25\right|,
$$

which does not depend on the gain (however, $G>1.3$ is required to ensure suppression of the lowest mode). It gives $D_{\text {min }} \simeq 0.22|3.2-\xi|=1.1-2.0$ at $\omega_{f} / \omega_{0} \simeq 400$ (present situation [2]), and $D_{\min } \simeq 0.22|4.8-\xi|=1.5-2.4$ at $\omega_{f} / \omega_{0}=\omega_{s} / \omega_{0}=588.1$.

\section{CONCLUSION}

It is shown that the transverse instability of a rectangular bunch can be described by the same dispersion equation as a coasting beam, if an effective beam coupling impedance is used instead of the standard one. Several methods to calculate the effective impedance are considered: integral equation for the beam dipole moment, corresponding series of equations for Fourier harmonics, or equivalent series for amplitudes of synchrotron multipoles. The last method is most universal and convenient for a numerical solution because corresponding matrix is approximately diagonal. This property allows one to use relatively small fragments of the matrix to calculate its eigenvalues including high order ones, starting from desirable number and scanning step by step the whole matrix. This also means that any eigenmode includes a rather small number of the synchrotron multipoles and has a narrow-band spectrum. In particular, it follows from this that the spectrum of high order eigenmodes includes only high frequencies which typically damp sufficiently rapidly to exclude long-range

TABLE I. Achievable recycler beam density and intensity.

\begin{tabular}{lcccc}
\hline \hline Chromaticity & $\mathrm{A}^{\mathrm{a}}$ & $\mathrm{B}^{\mathrm{b}}$ & $\mathrm{C}^{\mathrm{c}}$ & $\mathrm{D}^{\mathrm{d}}$ \\
\hline-2 & $0.3(150)$ & $1.1(550)$ & $1.5(750)$ & $8.1(4000)$ \\
-6 & $0.8(400)$ & $2.0(1000)$ & $2.4(1200)$ & $9.2(4500)$ \\
\hline \hline
\end{tabular}

${ }^{\mathrm{a} N o}$ damper.

${ }^{\mathrm{b}}$ Digital damper $35 \mathrm{MHz}$.

${ }^{\mathrm{c} D i g i t a l}$ damper $53 \mathrm{MHz}$.

${ }^{\mathrm{d}}$ Analog damper $18 \mathrm{MHz}$. 
interaction in the beam. Therefore the effective impedance of these modes is proportional to $B^{-1}$, and high-frequency collective effects depend only on local linear density of the beam (however, it is important that the density is constant within the whole bunch). Dependence of the effective impedance on $B$ is diverse for lower modes, but typically it increases at the bunch shortening. For example, effective resistive wall impedance $\propto B^{-1 / 3}$ for the most unstable mode.

Being applied to the Fermilab recycler, the theory predicts achievable beam density summarized in Table I [see Eq. (42) for the units]. Numbers in brackets are achievable beam intensities in units of $10^{10}$ at the phase volume $4 S_{\|} \times$ $6 S_{\perp}=70 \mathrm{eV}$-s $\times 7 \pi$-mm-mrad.

High-frequency related results should be valid for the multibunch regime as well, restricting parameters of any bunch. However, they cannot be applied to very short bunches when penetration of particles into the barriers becomes essential. Beam shaping before extraction ("mining") is an example of such a regime, when a multipulse rf wave form is generated without any space between the pulses. Then potential wells are triangular, and the results break down. The possibility must not be ruled out that the threshold decreases and instability appears at the mining, an effect which could explain the slow transverse emittance growth observed in the recycler at the mining [11].

[1] Gerry Jackson, FERMILAB-TM-1991, 1996.

[2] J. L. Crisp, M. Hu, and V. Tupikov, in Proceedings of the 2005 Particle Accelerator Conference, Knoxville, TN, USA, 2005, p. 1239.

[3] F. Sacherer, CERN-SI-BR-72-5, 1972.

[4] M. Blaskievicz, Phys. Rev. ST Accel. Beams 1, 044201 (1998).

[5] M. Blaskievicz, M. A. Furman, M. Pivy, and R. J. Macek, Phys. Rev. ST Accel. Beams 6, 014203 (2003).

[6] A. Burov and V. Lebedev, AIP Conf. Proc. 773, 350 (2005).

[7] V. Balbekov, in Proceedings of the 2005 Particle Accelerator Conference, Knoxville, TN, USA, 2005, p. 2567.

[8] V. Balbekov, FERMILAB-FN-0782-AD, 2006.

[9] A. Piwinski, DESY 94-068, 1994.

[10] V. Balbekov, FERMILAB-FN-0783-AD, 2006.

[11] L. Prost, S. Shemyakin, C. Gattuso, S. Pruss, M. Sutherland, and M. Hu, https://beamdocs.fnal.gov/ DocDB/0020/002035/001/113005/EmitGrowthUpdate. ppt, 2005. 